\section{TRAYECTORIAS ACADÉMICAS Y MIGRACIONES ALTAMENTE CALIFICADAS: UNA APROXIMACIÓN AL CASO DE LOS CIENTÍFICOS Y CIENTÍFICAS RETORNADOS A LA CIUDAD DE SANTA FE (2001-2015)}

\author{
ACADEMIC TRAJECTORIES AND HIGHLY \\ QUALIFIED MIGRATION: APROACH TO THE CASE \\ OF SCIENTIFICS RETURN TO SANTA FE CITY \\ (2001-2015)

\section{MARÍA NAZARET SERRA •} \\ Becaria postdoctoral del CONICET con sede en el de- \\ partamento de Sociología, Facultad de Humanidades y \\ Ciencias, Universidad Nacional del Litoral. \\ E-mail: mariaserra78@gmail.com
}

\section{Resumen}

El artículo analiza las trayectorias académicas de científicos y científicas migrantes que luego de regresar al país se insertaron en espacios institucionales en la ciudad de Santa Fe a través de la carrera de investigador del CONICET. Se utilizaron datos y estrategias de análisis cuantitativos y cualitativos para caracterizar estadísticamente los contextos y recorridos de los retornados. Se observó que, si bien son trayectorias lineales en lo referente a eventos y transiciones significativas, las principales diferencias son producidas por el género, los incentivos para el retorno y la experiencia académica previa en el lugar de reinserción.

\section{Registro bibliográfico}

SERRA, MARÍA NAZARET «Trayectorias académicas y migraciones altamente calificadas: una aproximación al caso de los científicos y científicas retornados a la ciudad de Santa Fe (2001-2015)», en: ESTUDIOS SOCIALES, revista universitaria semestral, año XXVIII, $n^{\circ} 55$, Santa $\mathrm{Fe}$, Argentina, Universidad Nacional del Litoral, juliodiciembre, 2018, pp. 27-55.

\section{Abstract}

The article makes the academic trajectories of migrant scientists who, after returning to the country, inserted themselves in institutional spaces in the city of Santa Fe by the scientific researcher career offered by CONICET. Data and strategies of quantitative and qualitative analysis were used, in order to characterize statistically the contexts and journeys of those who return. It was observed that, although they are linear trajectories with respect to events and significant transitions, the main differences are produced by gender, incentives for return and previous academic experience in the place of reintegration.

\section{Descriptores - Describers}

Trayectorias académicas / migración altamente calificada / género / Ciudad de Santa Fe / incentivos para la reinserción

Academic trajectories / highly qualified migration / gender / Santa Fe City / incentives to return

Recibido: 09/08/2017 Aprobado: 18/04/2018 


\section{INTRODUCCIÓN}

Las migraciones altamente calificadas han cambiado de fisonomía en las últimas décadas. Junto a su expansión en términos numéricos, se han diversificado los países de origen y destino, los tipos de flujos y las políticas que las regulan. En Argentina en particular, el retorno anual de científicos residentes en el exterior aparece inscripto en el marco del afianzamiento de políticas relativas a la ciencia y a la tecnología entre los años 2004 y 20I5. A partir del aumento de recursos estatales hacia el sector y la implementación de políticas públicas orientadas a su desarrollo, fue posible ampliar la base de personas dedicadas a las carreras científicas y motorizar el regreso de científicos argentinos resientes en el exterior. Santa Fe Capital, lugar elegido para esta investigación, forma parte de este proceso ya que es una de las ciudades por fuera de la Capital Federal (CF) que ha observado un número significativo de retornos en el período de estudio considerado.

La idea de este trabajo es explorar algunas características de las trayectorias académicas de científicos y científicas migrantes que luego de regresar al país se insertaron en espacios institucionales ligados a la ciencia en la ciudad de Santa Fe. Si bien los migrantes calificados se suelen clasificar en diferentes tipos (MAHROUM, I999), aquí se tendrán en cuenta sólo a estudiantes y a científicos/académicos, en tanto se considerará la trayectoria de quienes fueron a perfeccionarse al extranjero luego de haber finalizado sus doctorados en Argentina y la de quienes emigraron para desarrollar la etapa doctoral completa en otro país; los que retornaron al país para continuar con sus recorridos profesionales a través de incentivos estatales como los programas de repatriación y/o ingreso a la carrera de investigador del Consejo Nacional de Investigaciones Científicas y Técnicas (CONICET).

Para delimitar el problema, entendemos por trayectorias académicas de científicos retornados al encadenamiento de eventos y transiciones por diferentes esferas institucionales y de la vida (laborales, de estudio, familiares y vinculares) en el país y en el extranjero. Si bien dichos recorridos son percibidos por los científicos en forma reflexiva como «experiencias individuales» $\mathrm{y}$ «decisiones personales», aquí se asume la impronta social y política que las enmarca. De allí que serán analizadas de acuerdo con aquellos condicionantes y acontecimientos claves que las direccionan en tres momentos: antes, durante y después del retorno. 


\section{CIFRAS PARA ENMARCAR LAS TRAYECTORIAS ACADÉMICAS ESTUDIADAS}

En los años sucesivos a la crisis económico-política que atravesó Argentina en el 200I, las políticas dedicadas a la ciencia y a la tecnología dieron un giro. La inversión en el área como fracción del PBI, por ejemplo, pasó de ser 0,4\% en el 2004 a 0,63\% en el 20I5 (MINCYT 20I7). En esta línea, el número de investigadores y becarios con jornada completa aumentó de 2947I en 2004 a 52970 en 2015 (79,7 \% más), por lo que la relación entre cantidad de científicos dedicados a tiempo completo a la actividad científica y personas económicamente activas pasó de I,9/IOOO en 2004 a 3/IOOO en 20I2/20I5 (MINCYT, 20I7).

Este aumento de recursos económicos dedicados a la ciencia y la tecnología por parte del Estado nacional posibilitó no sólo que la actividad científica se convirtiera en un destino laboral más certero para muchos graduados, sino también el retorno y la movilidad de científicos argentinos resientes en el exterior. Santa $\mathrm{Fe}^{1}$ es una de las ciudades del país - por fuera de la provincia de Buenos Aires (Bs. As.) y de la $\mathrm{CF}-$ que presenció un mayor número de retornos en el período considerado, a través de la vinculación entre el CONICET, universidades nacionales y privadas y otros organismos estatales.

De acuerdo con los datos obtenidos, entre $200 \mathrm{I}^{2}$ y 2015 volvieron al país II6I científicos para ingresar a la carrera de investigador del CONICET. De la totalidad de los retornados, $55 \%$ se estableció en el área metropolitana ${ }^{3}$ y $21 \%$ eligió la región centro ${ }^{4}(\mathrm{RC})$, mientras que $8 \%$ se estableció en $\mathrm{Cuyo}^{5}$ y Patagonia ${ }^{6}$ (el mismo

\footnotetext{
${ }^{1}$ En lo que refiere al personal (investigadores, becarios, administrativos y técnicos) de CONICET en la ciudad, los datos muestran la misma tendencia, ya que aumentó un 75\% entre 2003 y 2013 en http://www.upcnsfe.com.ar/nota/36085-en-10-anos-aumento-un-75-por-ciento-la-planta-de-coniceten-santa-fe (consultado en noviembre de 2018).

2 Si bien la ley Raíces se sanciona en el año 2008 y el Ministerio de Ciencia, Tecnología e Innovación Productiva menciona el año 2004 como inicio de las repatriaciones (PROGRAMA RAícES, 2015), los científicos que aparecen en los boletines informativos del Programa como "repatriados» volvieron aproximadamente desde el 2001 -como es el caso de uno de los científicos que fueron entrevistados para esta investigación- hasta el 2015 -año de corte del presente análisis.

${ }^{3}$ Buenos Aires y Capital Federal.

4 Provincias de Córdoba y Santa Fe

${ }^{5}$ La Rioja, Mendoza, San Juan y San Luis.

${ }^{6}$ Chubut, La Pampa, Neuquén, Río Negro, Santa Cruz y Tierra del Fuego.
} 
porcentaje en cada una de las dos regiones), y $5 \%$ y $3 \%$ en NOA $^{7}$ y NEA ${ }^{8}$, respectivamente (PROGRAma Raíces, 20I5). Santa Fe, en tanto una de las tres ciudades más importantes que conforman la RC, observó a lo largo de esos años 4I retornos, frente a 58 de Rosario y 124 de Córdoba.

En cuanto a la distribución por sexo, a nivel nacional los varones representan $59,4 \%$ y las mujeres $40,6 \%$ de los retornados. En la comparación con la RC, estos porcentajes se mantienen con un leve aumento de los varones, $60,5 \%$ frente a $39,5 \%$ de las mujeres. Sin embargo, al interior de dicha región, el panorama es otro. Si bien en Córdoba y en Rosario los porcentajes no tienen grandes variaciones con los anteriormente mencionados, en Santa Fe las mujeres retornadas representaron sólo 31,7\% (Cuadros I y 2), es decir, cerca de diez puntos porcentuales menos que en las otras dos ciudades.

CUADRO 1:

RETORNO DE CIENTÍFICOS

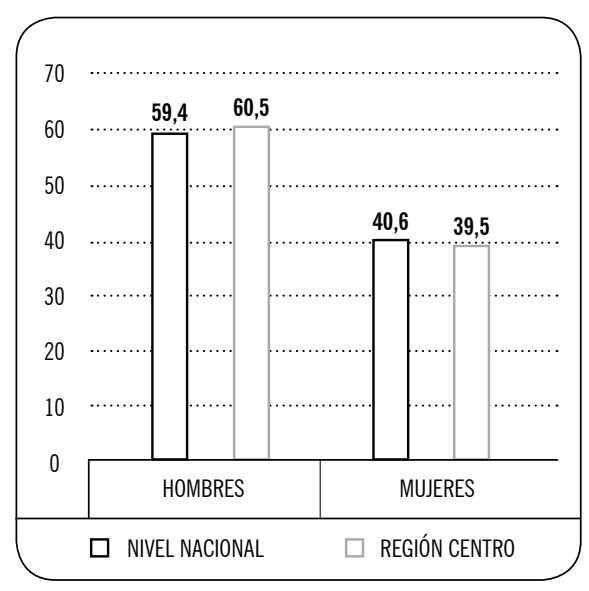

CUADRO 2:

RETORNO DE CIENTÍFICOS: REGIÓN CENTRO

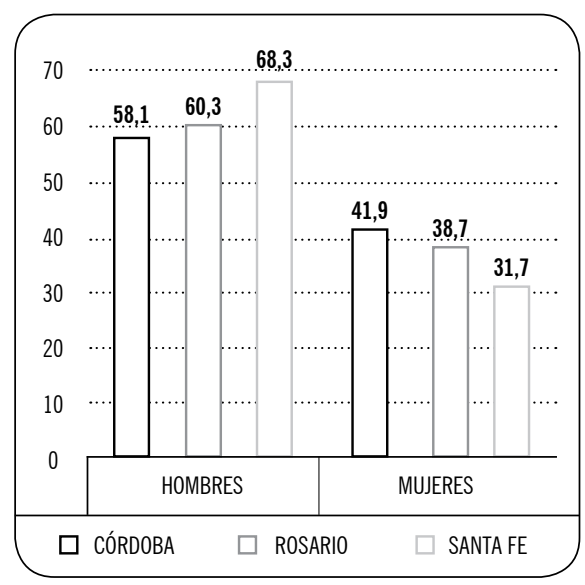

7 Catamarca, Jujuy, Salta, Santiago del Estero y Tucumán.

${ }^{8}$ Chaco, Corrientes, Entre Ríos, Formosa y Misiones. 
Los estudios sobre migraciones altamente calificadas muestran la creciente feminización de los flujos migratorios (LUCHILO, 2OII) y su vinculación con la mayor presencia de mujeres en la educación superior a nivel mundial (UNEsco, 2012). En Argentina, como parte de esta misma tendencia, del total de personas que completaron el nivel universitario, 54,5\% son mujeres (INDEC, 2OIO). Este dato coincide con la distribución por sexo en la matrícula de Universidad Nacional del Litoral de 20II, en la que las mujeres representaron 57\% (UNL, 20I4:42). Sin embargo, el número de mujeres migrantes altamente calificadas -en el caso de los retornos aquí estudiados- no sigue la tendencia antes mencionada, ya que la movilidad masculina sigue siendo más frecuente que la femenina. Más allá que en las últimas dos décadas el aumento de la presencia de mujeres en estos flujos haya sido mayor que el de los varones ${ }^{9}$ (LOZANO Y GANDINI, 2009), la incidencia del fenómeno de los migrantes altamente calificados es más significativo entre los varones que en las mujeres (dato que se intensifica en el contexto aquí estudiado).

En lo que refiere a las áreas de conocimiento ${ }^{10}$ de los retornados, existe una gran diferencia entre lo que sucede con las Ciencias Sociales (cs) ${ }^{11}$ a nivel nacional y regional. En el primer caso, las Cs ocupan el primer puesto de retorno junto con las Ciencias Biológicas y de la Salud (Св) ${ }^{12}$, con $28,3 \%$ de retornados, pero en la $\mathrm{RC}$ pasa a estar en cuarto lugar con $8,5 \%$. Al interior de la RC, por su parte, el poco peso de las Cs entre los retornados se profundiza en Santa Fe en tanto sólo 2,5\% pertenece al área. Otros porcentajes para destacar de la ciudad de Santa Fe se ligan

\footnotetext{
9 Según Lozano y Gandini (2009) entre 1990 y 2007 en los países OCDE el stock de migrantes de más de 24 años con más de 13 años de escolaridad creció $97 \%$ entre los varones y $127 \%$ entre las mujeres. ${ }^{10}$ CONICET divide las disciplinas en cuatro grandes áreas de conocimiento: 1) Ciencias Agrarias, Ingeniería y de Materiales, 2) Ciencias Biológicas y de la Salud, 3) Ciencias Exactas y Naturales 4) Ciencias Sociales y Humanidades (CONICET, 2017).

${ }^{11}$ El área de Ciencias Sociales incluye las siguientes disciplinas: Derecho, Ciencias Políticas y Relaciones Internacionales; Literatura, Lingüística y Semiótica; Filosofía; Historia, Geografía, Antropología Social y Cultural; Sociología, Comunicación Social y Demografía; Economía, Ciencias de la Gestión y de la Administración Pública; Psicología y Ciencias de la Educación; Arqueología y Antropología Biológica (CONICET, 2017).

${ }^{12}$ El área Ciencias Biológicas y de la Salud incluye las siguientes disciplinas: Ciencias Médicas, Biología, Bioquímica y Biología Molecular y Veterinaria.
} 
a la importancia que tienen las Ciencias Agrarias, Ingeniería y de Materiales (CA $)^{13}$, en tanto ocupan el primer puesto con $36,5 \%$ frente al tercer puesto en el que se ubica tanto en las otras dos ciudades de la misma región como a nivel nacional. Finalmente, resalta el lugar de la Tecnología $(\mathrm{T})^{14}$, ya que llegan a ser casi $5 \%$, frente a $0,6 \%$ del nivel nacional (Cuadros 3 y 4 ).

En cuanto a los continentes de los que han regresado los científicos estudiados, América ocupa el primer lugar a nivel nacional con 53\% (como se verá luego, principalmente por los retornos de Estados Unidos) y, en un segundo lugar, Europa con $45 \%$. Estos datos se invierten en el contexto regional, ya que Europa pasa a tener $55,4 \%$ de los retornados y América 43,3\%. Estas diferencias se intensifican en la ciudad de Santa Fe (incluso respecto de Rosario y Córdoba) ya que 65,9\% volvió de Europa y sólo 34,I \% de América (Cuadros 5 y 6).

En cuanto a los países elegidos por los retornados como destino para realizar la experiencia académica por fuera del país, Estados Unidos y España ocupan el primer y el segundo puesto en cualquiera de los niveles de análisis. Sin embargo, a nivel nacional el tercer lugar lo ocupa Francia y el cuarto Alemania, mientras que, a nivel regional esa relación se invierte a favor de Alemania. En lo que respecta a los casos santafesinos, Alemania y España ocupan el segundo lugar y se observa no sólo una disminución de la preeminencia de Estados Unidos sino también una distribución más homogénea de los retornos entre países como Francia, Brasil, Reino Unido, entre otros (Cuadros 7 y 8).

${ }^{13}$ El área Ciencias Agrarias, Ingeniería y de Materiales incluye las siguientes disciplinas: Ciencias Agrarias; Ingeniería Civil, Mecánica, Eléctrica e Ingenierías relacionadas; Hábitat, Ciencias Ambientales y Sustentabilidad; Informática y Comunicaciones; Ingeniería de Procesos, Productos Industriales y Biotecnología; Desarrollo tecnológico y Social y Proyectos Complejos (CONICET, 2017).

${ }^{14}$ Tecnología no es una de las cuatro áreas principales de CONICET. Sin embargo, la institución la asume como área de conocimiento a la que pertenecen los retornados -de cualquier disciplina- que a partir de sus investigaciones desarrollaron algún producto y lo patentaron. 
CUADRO 3:

RETORNO POR ÁREA DE CONOCIMIENTO

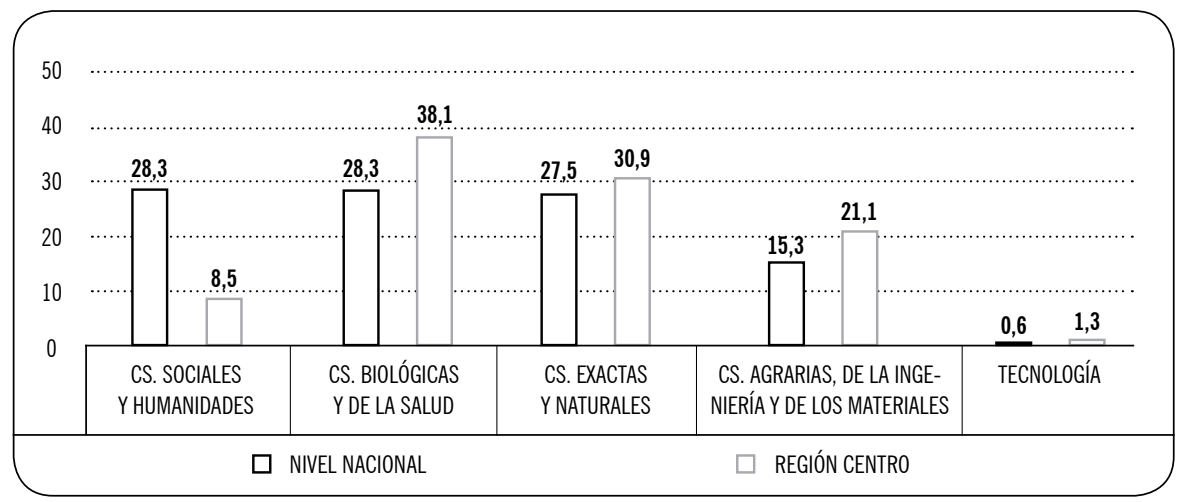

CUADRO 4:

RETORNO POR ÁREA DE CONOCIMIENTO: REGIÓN CENTRO




CUADR0 5:

CONTINENTES DE RETORNO

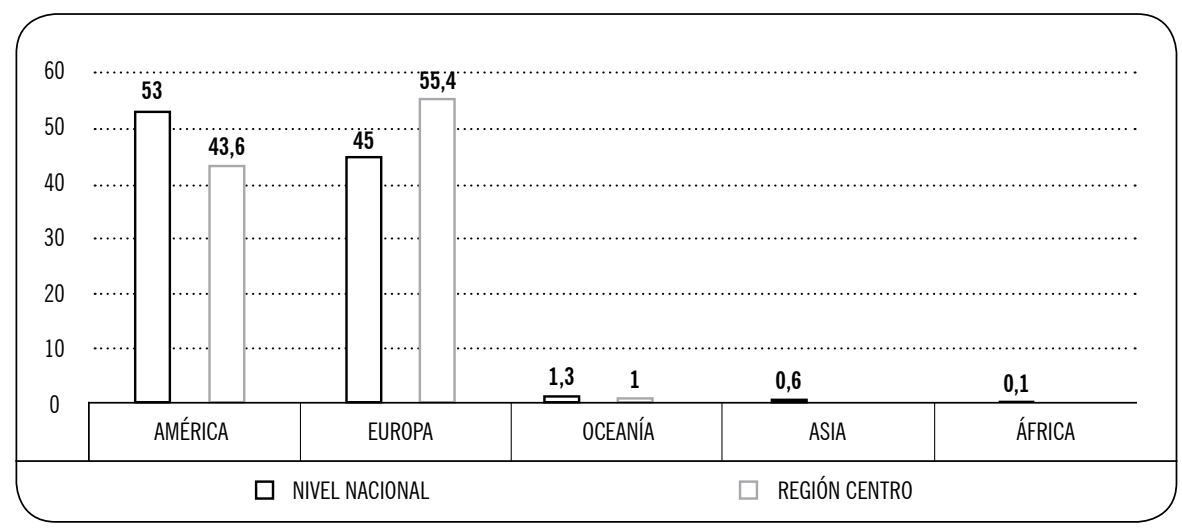

CUADRO 6:

CONTINENTES DE RETORNO: REGIÓN CENTRO

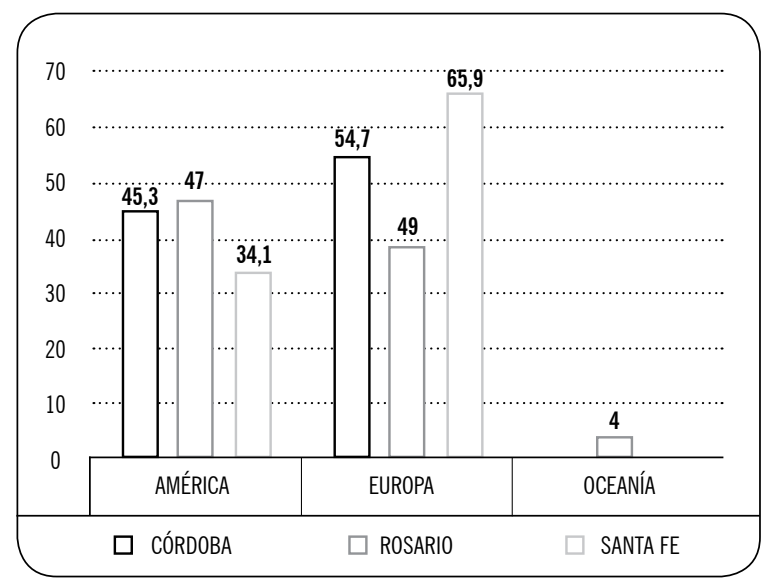

34 MARÍA NAZARET SERRA [Trayectorias académicas y migraciones altamente calificadas] [pp. 27-55] 
CUADRO 7:

PRINCIPALES PAÍSES DE RETORNO

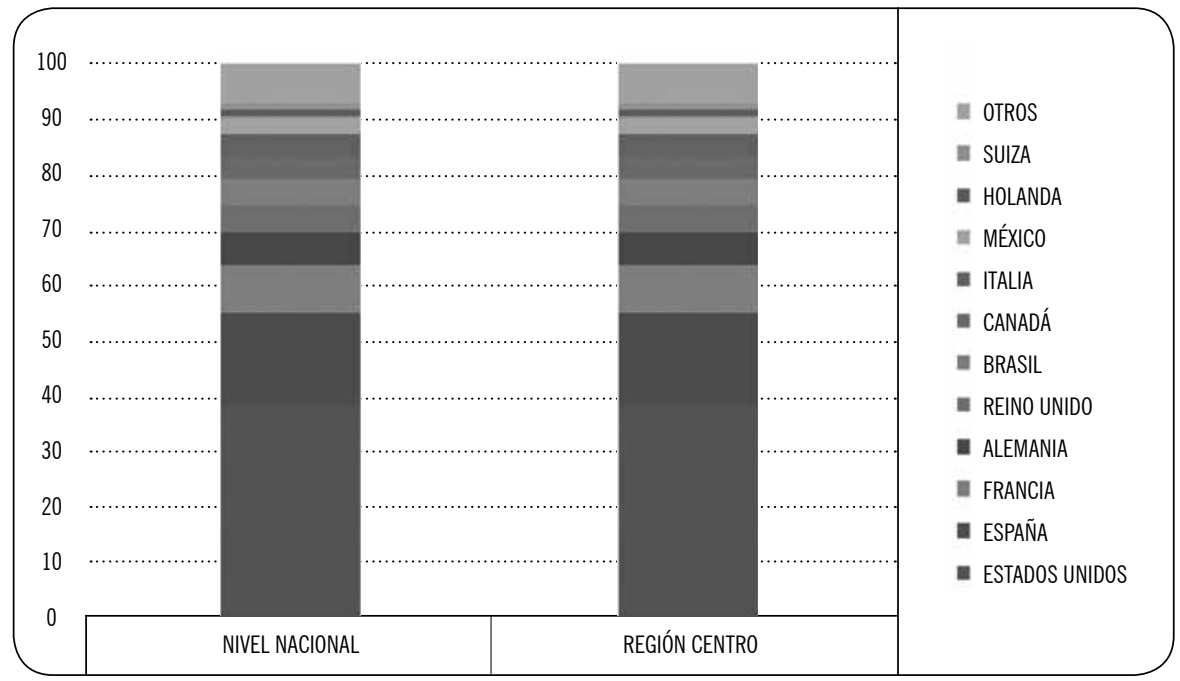

CUADRO 8:

PRINCIPALES PAÍSES DE RETORNO: REGIÓN CENTRO

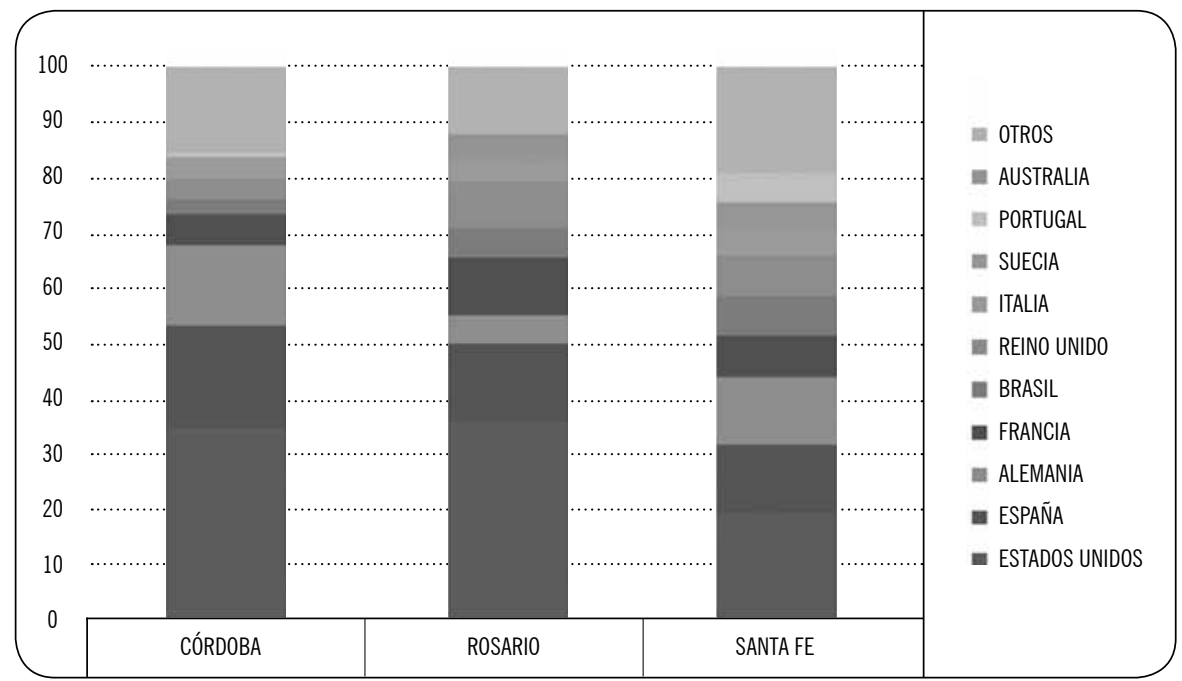




\section{PRECISIONES METODOLÓGICAS}

Para aproximarnos al caso (COLLER, 2005; STAKe, 1995) de los científicos y las científicas retornados a Santa Fe a través incentivos estatales (200I-20I5), se siguieron dos estrategias metodológicas. La primera cuantitativa -desarrollada en el apartado anterior- en la que utilizamos datos cuantitativos con el fin de caracterizar y establecer generalizaciones estadísticas acerca del contexto en el que se encuentran los científicos retornados a la ciudad de Santa Fe. Para ello, seleccionamos porcentajes producidos por el MINCYT vinculados a los científicos retornados a nivel nacional. Luego, cargamos, procesamos y analizamos los datos relativos a la RC y Santa Fe, y, por último, incorporamos otro tipo de información (como censos y registros de instituciones universitarias).

En lo que refiere a la estrategia cualitativa, nos centramos en el método biográfico (SAUTU, I999) con el fin de realizar una inmersión inicial en el campo (SAMPIERI, 20I4) de las trayectorias académicas (eventos y transiciones) de los científicos retornados. Para ello realizamos nueve entrevistas en profundidad -entre febrero de 2016 y marzo de 20I7- a científicos y científicas que residen en Santa Fe luego de una estadía de estudio y/o laboral en el extranjero y dos a autoridades de CONICET en ese momento. De las once entrevistas, diez las llevamos a cabo en los lugares de trabajo de los investigadores y solo una de forma virtual ${ }^{15}$.

De los 4I retornados a Santa Fe, decidimos construir una muestra dirigida en base a dos criterios o casos tipo (SAMPIERI, 20I4) que se consideraron condicionantes de las trayectorias estudiadas. El primero ligado al tipo de recorrido de estudio que se fue a hacer a otro país (doctorado completo o posdoctorado) y el segundo al género de los entrevistados. En un caso debido a que el tipo de experiencia académica elegida condiciona las oportunidades/pérdidas laborales y académicas aquí y allá, los tiempos de permanencia en el extranjero y los vínculos establecidos en ambos lugares (PELLEGRINO, 20OI; FLORES, 2OII). Y, en el otro, en tanto se asume que el género delimita no tanto el ingreso al mundo científico como el modo y las posibilidades de desarrollar una carrera profesional (HOCHSCHILD, 2OI3).

${ }^{15} \mathrm{Al}$ momento de la realización de la entrevista, el investigador de Ciencias Sociales y Humanidades se encontraba realizando una estadía de seis meses en una universidad de Estados Unidos. Como consecuencia de ello, la entrevista se llevó a cabo vía Skype. 


\begin{tabular}{|l|c|c|}
\hline \multirow{2}{*}{ EN EL EXTRANJERO } & \multicolumn{2}{|c|}{ SEXO } \\
\hline & $\mathrm{F}$ & $\mathrm{M}$ \\
\cline { 2 - 3 } DOCTORADO & 1 & 2 \\
POSDOCTORADO & 2 & 4 \\
\hline
\end{tabular}

Si bien los criterios centrales para la selección de la muestra fueron los antes mencionados, también tuvimos en cuenta las áreas de conocimiento a la que pertenece cada uno para no perder de vista la relación que pudiera llegar a existir entre las diferentes áreas y las trayectorias estudiadas. Cabe mencionar que la ausencia de mujeres en el área Tecnología y cs se debe a que hasta la fecha de realización del trabajo de campo no había ninguna investigadora perteneciente a las mismas que haya regresado a Santa Fe.

\begin{tabular}{|l|c|c|}
\hline \multirow{2}{*}{ ÁREA DE CONOCIMIENTO } & \multicolumn{2}{|c|}{ SEXO } \\
\hline & $\mathrm{F}$ & $\mathrm{M}$ \\
\cline { 2 - 3 } CIENCIAS EXACTAS Y NATURALES (CE) & 1 & 1 \\
CIENCIAS AGRARIAS, DE LA INGENIERÍA Y DE LOS MATERIALES (CA) & 1 & 1 \\
TECNOLOGÍA (T) & - & 1 \\
CIENCIAS SOCIALES Y HUMANIDADES (CS) & - & 1 \\
CIENCIAS BIOLÓGICAS Y DE LA SALUD (CB) & 1 & 2 \\
\hline
\end{tabular}

\section{CONCEPTOS PARA ENTENDER LAS TRAYECTORIAS ACADÉMICAS DE LOS CIENTÍFICOS Y CIENTÍFICAS MIGRANTES}

Hemos elegido abordar este trabajo desde dos perspectivas teóricas diferentes pero complementarias. Una relativa a los movimientos migratorios de personas altamente calificadas y la otra a las trayectorias académicas. En el primer caso, para identificar las características y particularidades histórica de dichos flujos. En el segundo, para entender los recorridos de los científicos aquí estudiados. Recorridos que expresan un conjunto de eventos y transiciones condicionado no sólo por el contexto socio histórico que lo enmarca, sino también por disposiciones y posiciones ligadas al género, al tiempo (individual e histórico) y a los espacios institucionales en los que se desarrollan. 
Los movimientos migratorios de científicos son menos dependientes de los ciclos económicos, lo cual no significa que sean indiferentes a las coyunturas de crisis sino a que la decisión de migrar tiene mayor probabilidad de estar asociada al encuentro de una ocupación acorde con el nivel de formación del potencial migrante (LUCHILO, 2007). Es por ello que estas migraciones suelen ser consideradas "privilegiadas», en tanto incluye a «actores sociales que forman parte de un movimiento legitimado» (MERA Y HALPERN, 2OII:IOO) caracterizado por tener facilidades a la hora de integrarse económica, social y culturalmente en el contexto de arribo.

No todas las migraciones altamente calificadas se encuentran vinculadas al ámbito académico. De acuerdo con la clasificación elegida para este trabajo, además de las académicas, existen otras asociadas a empresas. Éstas, a diferencia de las aquí estudiadas, refieren a aquellos actores que migran como una forma de buscar un incremento de su capital (empresarios) o bien como consecuencia de decisiones de negocios de las empresas en las que trabajan (gerentes y ejecutivos). Por otro lado, se encuentran los ingenieros y técnicos quienes forman parte de otro de los grupos asociados a este tipo de migraciones. Éstos se caracterizan por moverse influenciados por las posibilidades laborales que se abren en otros países. Por último, los estudiantes, académicos y científicos -a cuyo análisis se abocan en estas páginas- se mueven en el marco de las necesidades y estrategias de las universidades ${ }^{16}$ a las que se encuentran vinculados, como así también de la valoración que hacen de lo que puede aportar a la propia carrera -en cuanto a contactos y reputación- establecerse en un espacio académico de otro país (MAHROUM, I999).

La direccionalidad de este tipo de migración, ya no se centra en el pasaje SurNorte propio de las décadas de 1960 y 1970 sino que, por el contrario, ha pasado a ser multilateral y policéntrica, tal como lo demuestra el intercambio entre Argentina y Brasil mencionado anteriormente. Igualmente, más allá de este giro, los movimientos no llegan a ser del todo multidireccionales, pues los flujos se siguen dirigiendo con mayor frecuencia de los lugares menos desarrollados a los sitios más competitivos de la economía mundial del conocimiento (MEYER ET AL., 20OI). Si bien algunos estiman que luego de la crisis económica iniciada en el 2008 esta tendencia comenzó a disminuir (PAPADEMETRIOU ET AL., 2OIO), los cambios en el mediano plazo no se observan en forma marcada (algunos de los aquí entrevistados, ${ }^{16}$ En el caso argentino se incluye al Ministerio de Ciencia, Tecnología e Innovación Productiva y sus
organismos dependientes. 
por ejemplo, migraron luego de ese año). Una de las explicaciones posibles se asocia al hecho de que los procesos migratorios tienen un componente de perpetuación y de «causación acumulativa». Es decir, los mismos desarrollan mecanismos de dependencia de trayectoria que hacen que la migración se mantenga o aumente a pesar de que existan obstáculos importantes para el migrante y su familia como el alto desempleo o políticas de admisión más restrictivas (LUCHILO, 20II:37).

En lo que refiere a la lectura de la relación entre las migraciones calificadas y ciencia local/nacional, no necesariamente se construye en términos de pérdidas. Como afirma PELlegrino (200I: 13I) «el debate sobre la fuga de cerebros (brain drain) ha sido progresivamente sustituido por propuestas para estimular la circulación y el intercambio de cerebros (brain circulation y brain exchange), con el propósito de superar o compensar las pérdidas debidas a la emigración. En estas propuestas, el énfasis se pone en la movilidad y el intercambio de recursos altamente calificados entre los países de origen y los desarrollados». Sin embargo, desde otra perspectiva, se asume que este intercambio o integración, lejos de establecerse como una relación entre "pares», tiende a ser subordinada. En la medida en que, por ejemplo, la elección de las líneas de investigación, la visión de conjunto de los problemas conceptuales y, también, sus utilidades reales o potenciales se producen con una fuerte dependencia de los dictados operados por los centros de referencia, localizados en los países más desarrollados (KREIMER, 2006).

En lo que respecta a las trayectorias académicas aquí narradas, si bien aparecen con una fuerte impronta individual ("quería hacer la experiencia en el extranjero», "consideraba que era bueno para mi carrera»), éstas son colectivas. Los «individuos no se desplazan al azar por el espacio social», afirma BOURDIEU (2002:IO8), lo que implica asumir que las trayectorias de los científicos estudiados se encuentran condicionadas tanto por sus disposiciones y posiciones de origen (género, clase social, preferencias de estudio, etc.), como por acontecimientos colectivos (crisis económicas, cambios en el sistema de Ciencia y Técnica, por ejemplo) o personales (elecciones de pareja, nacimientos, separaciones, etc.), los que, en tanto tales, pueden dar lugar a puntos de inflexión (SAUTU, 1999) y evidencian la necesidad de considerar diferentes aspectos de la propia experiencia para poder entender estos recorridos.

Una trayectoria «objetiva» puede ser entendida como una secuencia de posiciones sociales ocupadas en diferentes esferas de la vida, medidas por categorías estadísticas o condesadas en una tendencia general. Se trata de un modelo de relativa estabilidad y cambio a largo plazo, como estado de inserción de papeles 
que representan variación a lo largo del tiempo. Una transición -como parte de dicha trayectoria- da cuenta de un cambio al final de un estado y un evento es lo que ocurre en un cierto tiempo y lugar. Los eventos pueden ser normativos (graduaciones, nacimientos, matrimonios) y no normativos (crisis económicas, separaciones, retorno) (DUBAR, I998; ABвOTT, 200I; VIDEGAIN, 20I5). En estas páginas, los primeros están asociados a un tiempo normativo, es decir, aquel que rige ciertos comportamientos y acciones por fuera del cual se asume que existe un «problema» o «desfasaje». Los segundos, por su parte, se vinculan con un tiempo histórico, es decir circunstancias ligadas al acontecer social, así como por un tiempo individual, reconocido en aquellos sucesos personales que direccionan la trayectoria y que pueden estar más o menos cercanos a la norma antes referida.

La profesión académica tradicional se basa en una cadena de experiencias ligadas a: a) trabajar compitiendo con los demás; b) obtener reconocimiento y hacerse una reputación durante la juventud; c) dedicarle todo el tiempo posible a tal empresa; d) minimizar la vida familiar y dejarla en manos de otros (HOCHSCHILd, 2OI3). En términos de BOURDIEU ([1976] I994), podría traducirse en el trabajo que un investigador tiene desarrollar a lo largo de su vida laboral para poder acumular capital científico -además de capital social, cultural y económico (BOURDIEU, 2000)- y las tareas ligadas al ámbito doméstico que debe evitar para que esto sea posible.

Según HOCHSCHILD (2OI3: 348), «toda carrera comprende una serie de posiciones y logros que se miden según parámetros estrictos y competitivos en comparación con otras carreras, de modo tal que cuentan hasta las diferencias minúsculas entre los logros de cada una». Y, en este recorrido de acumulación de capital científico, el tiempo en el que se producen ciertos eventos ocupa un papel central. No es lo mismo finalizar la licenciatura a los 30 años que a los 23, ni el doctorado a los 30 años o a los 36. De hecho, quienes no cumplen el tiempo normativo establecido por cada disciplina -y en el caso específico argentino establecido por CONICETlo reconocen como un desfasaje que se trata de acomodar (de ser posible) o bien como una marca que resta capital científico al propio recorrido.

\section{ANTES DE PARTIR}

La mayoría de los científicos aquí entrevistados hicieron sus estudios de grado en universidades estatales, en tanto sólo uno estudió en una universidad privada. 
En lo que refiere a la ciudad, cuatro estudiaron en Santa Fe -tres en la Universidad Nacional del Litoral (UNL) y uno en la Universidad Católica (UCSF)-, y el resto en Córdoba, Entre Ríos, Salta y Buenos Aires. La edad que tenían los entrevistados al obtener el grado oscila entre los 23 y los 25 años. De los seis que concluyeron el doctorado en Argentina, cuatro lo hicieron en UnL, uno en la Universidad de Buenos Aires y otra en la Universidad Nacional de Rosario, con entre 27 y 3 I años. Frente a la pregunta por los motivos de la decisión de migrar, la mayoría de los entrevistados vincularon su respuesta al desarrollo de la propia carrera científica (conocer qué y cómo se investiga en otros lados, «enriquecerse» en términos de experiencia de vida y profesional, conocer otras culturas, o viajar). Sin embargo, los que emigraron entre los años 2000 y 2002 reconocieron que el contexto inestable a nivel económico por el que atravesaba Argentina incidió en la búsqueda de horizontes laborales fuera del país. «En esa época podías ser Einstein y no entrar a carrera [de investigador de CONICET]. Era natural irse, ahora el camino es diferente... ${ }^{17}(\mathrm{M}, \mathrm{PE}, \mathrm{CE})$, expresaba una de las entrevistadas que migró en ese período como síntesis de las razones de su partida, mientras que otro relata:

«Tuve una beca de CONICET para empezar un doctorado, lo empecé, pero después vino el corralito, De la Rúa, estaba todo mal, no había trabajo, todo era un desastre y ahí fue cuando decidí irme al exterior. Había hecho un año y medio más o menos del doctorado (...) Estaba muy rara la situación. En principio veía que no había mucho futuro en Argentina (...) Entonces me fui a EE.UU. Hice casi dos años de doctorado acá y me fui en el 2002» (H, DE, CE).

Es de destacar -en función de los criterios de selección de casos- que la decisión de migrar para continuar la carrera académica presenta diferencias entre los varones y mujeres que al momento de la partida se encontraban en pareja. En el primer

\footnotetext{
${ }^{17}$ Al respecto, la directora de CONICET Santa Fe asumía «De la misma forma que te voy a decir algo que no estás mirando, hay muchos que no se quieren ir. Uno de los problemas hoy en la formación en las áreas biológicas (no te voy a hablar de otras porque a estás las conozco bien) (...). Cuando yo me fui no se podía ingresar a carrera si uno no tenía un posdoctorado en el exterior. Ahora, en los últimos años, no puedo decir cómo será de a hora en más, pero de acuerdo a lo que puedo mirar de ahora para atrás, entró muchísima gente a carrera de investigador sin tener posdoctorado en el exterior (...) Con lo cual ha crecido en la comunidad mucha gente que no tiene esa formación que es muy enriquecedora, es decir que les falta eso» (entrevista realizada en febrero de 2016).
} 
caso, las mujeres acompañaron la decisión de su pareja y lograron acomodar sus actividades (estudios, trabajos, etc.) en el lugar por esta elegido para continuar con su recorrido académico. Esto responde a lo que Hochschild llama «perfil clásico del hombre académico» hecho a la medida de un hombre tradicional con una esposa tradicional (HOCHSCHILD, 20I3: 337):

«-¿Tu mujer es científica?

-No, ella es abogada. Ella se fue sin ningún tipo de conocimiento del alemán. Estuvo estudiando alemán durante un año y medio (...) Sin ese examen no podés entrar a la universidad. Ella hizo ese examen y le fue muy bien y después hizo un Máster en Derecho comparado» (H, DE, CS).

-«En el instituto ella trabajaba en una cosa que le dicen (...) ayudante técnico, me ayudaba a mí. Mi jefe la contrató para que me ayudara a mí en mi investigación. Hacía papelerío que había que hacer, e incluso me ayudaba con experimentos. A ella le vino bien para no tener que estar tanto tiempo encerrada y sola» (Н, РЕ, Св).

Las mujeres científicas, a diferencia de los varones, migraron con su pareja, quien también es científico, a un lugar consensuado en términos de intereses de investigación. A lo largo del trabajo de campo no apareció ningún tipo de mención, ni siquiera como experiencia conocida, de mujeres que hayan llevado a su pareja y que ésta se acomode a sus elecciones laborales o de estudio.

«-Él se va en noviembre [a Holanda] comienza el primero de diciembre su posición allá. Y yo justo, mientras él se iba, yo estaba entregando mi tesis en posgrado. Él se va y nos dijimos, como yo presenté la tesis ahora empiezo a buscar algo. Ahí sí se concretaba la idea de irnos afuera, pero yo estaba un poquito más acotada por el lugar de trabajo.

- ¿Vos tenías que buscar algo en Holanda?

-Exactamente así fue» (M, PE, CA).

Entre los entrevistados, algunos hicieron el doctorado en Argentina con la beca de CONICET. Entre ellos, los que se fueron al extranjero antes del 2002 a realizar una estadía posdoctoral, lo hicieron con las becas externas que otorgaba el organismo hasta esa fecha. En cambio, los que migraron desde el 2003 en adelante solventaron su estancia con distintos tipos de financiamiento (becas o contratos 
laborales temporarios) con la diferencia de que en esos casos no tenían la obligación de volver al país como sí ocurría con los primeros. Sin embargo, ambos estaban convencidos de volver a la hora de partir, los primeros ligados a una obligación y, los segundos, debido a que contaban con la posibilidad clara de seguir con la carrera de investigador que les habilitaba CONICET.

«-Hay una cosa que soy, y eso no me da vergüenza decirlo, soy muy prolijo y ordenado. Yo tenía la obligación de volver y solo de pensar en mi padre que fue el que me hizo así. Si vos firmaste que volvías, tenés que volver. En todo caso estás un tiempo y después te volvés. Quiero decir, hay algo de eso también (H, PE, T)» ${ }^{18}$.

«-Es cierto que cuando uno se va no se va pensando en irse a vivir afuera, siempre se va pensando en ir a hacer una etapa, entonces pasado 4 o 5 años se cumple ese ciclo. $\mathrm{Y}$ uno ya no se fue con la idea de quedarse para siempre, entonces la idea de volver ya está antes de irse» $(\mathrm{H}, \mathrm{PE}, \mathrm{CB})^{19}$.

A diferencia de los anteriores, los que decidieron hacer el doctorado afuera son aquellos que emigraron sin una idea clara de retorno. Si bien los tres eran becarios del CONiCET, optaron por abandonar la carrera en Argentina y empezar de cero en otro país y espacio académico. La continuación de la actividad académica en el exterior se presentaba no sólo como una oportunidad para evitar un futuro incierto, sino también como una estrategia posible -gracias a su corta edad- para abrirse a nuevos horizontes de investigación: ir más allá de los grupos de investigación locales y conocidos, conocer qué se investiga en otros lugares, entre algunas de las razones mencionadas.

«-El detonador fue la situación del país, pero fue un conjunto digamos. Por ahí necesitaba salir de la endogamia de acá, era joven y dentro de las posibilidades que se abrieron aproveché [...] fue justo antes del corralito y todas esas cosas, después habían devaluado, la gente estaba sin trabajo en la calle; eso, por un lado, la situación social y por el otro que cuando uno es joven le gusta pensar en la posibilidad de ver qué hacen en otros lugares» (H, DE, $\mathrm{CE})$.

\footnotetext{
18 Inició su estadía posdoctoral en 1999 y retornó en 2001.

19 Inició su estadía posdoctoral en 2008 y retornó en 2013.
} 
En lo que refiere a los capitales cultural y científico, los que se fueron a hacer el doctorado completo no sólo habían alcanzado el título de grado como máximo a los 23 años, sino que ya contaban con una cierta trayectoria docente y de investigación en el lugar de partida (los tres eran becarios doctorales del CONICET). Por su parte, quienes estuvieron en el extranjero en una instancia posdoctoral, ya habían logrado un cierto recorrido en lo que refiere a publicaciones, lo que algunos conjeturan motivo de la aceptación de la candidatura.

«-Publiqué muchísimo como doctorando acá. Me fue muy bien en el doctorado. Siempre publiqué en revistas internacionales [...] creo que eso hizo que a la respuesta me la dieran a la semana siguiente. Era un buen candidato» (H, PE, CA).

Finalmente, el país elegido para hacer el doctorado o el posdoctorado coincide con los destinos privilegiados observados en la caracterización general de los movimientos internacionales de científicos en Santa Fe. Con excepción de una investigadora que estuvo en Holanda, el resto migró hacia Estados Unidos, España, Alemania, Francia y Brasil. La elección de Estados Unidos aparece vinculada a la idea de que allí se encuentran los investigadores «de avanzada» (M, PE, CE) y los «mayores especialistas» ( $\mathrm{H}, \mathrm{PD}, \mathrm{CA})$ de sus propias áreas temáticas, mientras que, en relación a Francia y Alemania, emerge el interés por «una tradición» ( $\mathrm{H}$, $\mathrm{PE}, \mathrm{T}$ ) y una «cultura» ( $\mathrm{H}, \mathrm{PD}, \mathrm{CB}$ ) (más allá de la materia específica a la que se pertenece). Por su parte, España se asocia a un contexto menos «cerrado» (H, PE, Св) en términos culturales e idiomáticos que otros países europeos y, Brasil, como un lugar «cercano» ( $\mathrm{M}, \mathrm{DE}, \mathrm{CB})$.

En síntesis, los científicos que hicieron el doctorado en Argentina se fueron, en general, con una idea de retorno más clara que los que emigraron para hacer el doctorado completo en otro país. Sin embargo, el contexto económico de crisis de 200I condicionó a quienes - de ambos grupos- en ese momento se encontraban en la búsqueda de otros horizontes por fuera de Argentina para perfeccionarse. En lo que refiere al género, las mujeres entrevistadas decidieron migrar con sus parejas-que también eran científicos-a un lugar consensuado y conveniente para ambos en términos de sus carreras. Mientras que, para los varones entrevistados, la situación fue diferente, en tanto partieron acompañados por sus parejas y con la expectativa a futuro de que ellas lograran acomodar sus actividades laborales y/o de estudio al lugar elegido. 


\section{MIGRACIÓN Y EXPERIENCIA ACADÉMICA EN EL EXTRANJERO}

Como se mencionó anteriormente, de los nueve científicos, seis emigraron para transitar una etapa posdoctoral luego de finalizar el doctorado en Argentina, mientras que los otros tres lo hicieron para hacer el doctorado completo. Los que se fueron a hacer el posdoctorado con posterioridad al 200 I estuvieron entre 3 y 6 años en el extranjero, mientras que los que lo hicieron antes no más de tres años (dado que con las becas externas del CONICET era obligación el retorno pasado ese período de tiempo). A diferencia de éstos, los que realizaron el doctorado en su totalidad en el extranjero, permanecieron entre 7 y II años en el mismo país para hacer el posdoctorado y/o continuar trabajando como profesores universitarios una vez concluido el ciclo doctoral. La edad de este grupo al finalizar dicho ciclo se sitúa entre los 32 y 36 mientras que los del grupo antes caracterizado como máximo llegaba a los 3I años. Esta diferencia se repite a la hora del retorno, ya que unos volvieron entre los 29 y los 36 años y los otros alcanzaron los 40 años en ese momento.

De los entrevistados, los que transitaron todo el doctorado en el extranjero no sólo consideraron la posibilidad permanecer en el mismo país del doctorado u en otro, sino que hicieron el intento o se quedaron a trabajar un tiempo.

«-¿En algún momento pensaste en quedarte a vivir afuera?

-Sí, no es algo que haya tenido decidido te digo la verdad. En algún momento surgió la posibilidad de venirme a Estados Unidos. No estaba seguro de venirme [a Estados Unidos] para ser sincero por un montón de motivos, personales, afectivos (...) Y en el ínterin me surgió la posibilidad de repatriación y me fui a Argentina. Contestando a tu pregunta pensé en esa posibilidad, no sé qué hubiera pasado si me hubiera salido la opción esa en Estados Unidos» (H, DE, CS).

«-Sí, estuve viviendo un tiempo allá cuando terminé el doctorado, estuve trabajando como 5 años (...) cuando estaba trabajando allá en principio no había razón para no quedarme...» (H, DE, CE).

Las visiones acerca del aporte que generado a su carrera científica el hecho de haberse ido se puede asociar a los capitales adquiridos e incorporados. En lo que refiere al capital económico sólo uno de los entrevistados-quien migró no sólo con la beca externas que hasta el 200 I otorgaba CONICET, sino también con la de la institución de acogida- manifestó que la experiencia académica tuvo sus frutos económicos: 
«-O sea que yo durante dos años dupliqué la beca, fueron años de grandísimos ahorros para mí porque fueron años en los que el peso estaba ultra devaluado. Vivíamos bien y además pudimos ahorrar. Estuve tres años, dos con beca de CONICET y en el 2003 ingreso a carrera, pero no me daban el ingreso. Como no se hacía efectivo el ingreso, en ese momento decido bueno me quedo un año más en Francia solo con el pago francés» (H, PE, CA).

El capital científico, al momento de la migración, está asociado a la posibilidad de hacer carrera, de dedicarle todo el tiempo posible a la actividad y de contar con mayores y mejores recursos para hacer trabajo científico.

«-Tenía todos los recursos disponibles, eso producto del laboratorio. Toda la gente capacitada que me ayude a sacar adelante las cosas que no necesariamente eran mi especialidad, sectores administrativos que se encargan de todo. Fueron cinco años en donde yo no me preocupé por conseguir plata y no me preocupaba por otra cosa que no fuera pensar y hacer ciencia. Y eso creo que me dio muchos frutos» ( $\mathrm{H}, \mathrm{PE}, \mathrm{CB})$.

«-La infraestructura que tienen no tienen comparación (...) Bueno lo que son las bibliotecas de las universidades alemanas son impresionantes. Es muy difícil no encontrar un libro o un artículo en una universidad alemana» (H, DE, CS).

"-No te voy a decir que quedé deslumbrado por el nivel que encontré allá, sí quedé deslumbrado con el hecho de que lo que allá simulaban después lo fabricaban. Tenía una aplicación» (H, PE, CA).

La decisión de volver aparece ligada a un evento pensado antes de la partida en el caso de los que se fueron hacer el posdoctorado, mientras que, para los que hicieron el doctorado, la idea del retorno presenta mayores vaivenes. Todos los entrevistados mencionaron a los afectos (familiares y de amigos) y algunos hicieron referencia a eventos significativos (separaciones o nacimientos) como motivos que incidieron en el retorno. Sin embargo, lo central está asociado a los incentivos para retorno y a las posibilidades que ello eventualmente reportaba a la propia actividad. Algunos consideraron que aquí tenían algo para dar que en el extranjero no era valorado como en el país, otros asumían haber concluido un ciclo y que tenían mejores posibilidades si regresaban y otros, en cambio, era el único lugar que les garantizaba un trabajo más estable. 
«-Por cuestiones personales, cuestiones familiares y también interiormente sentía que necesitaba un cambio. Allá era todo muy ordenado. Yo sentía que yo tenía algo para dar y que allá no iba a ser valorado. Sin embargo, acá en Argentina como que se necesitaba más» (H, DE, CE).

«-Uno da el salto para venir acá, al final de cuentas el objetivo final que uno se había planteado desde la carrera. Quiero hacer una carrera académica porque quiero tener mi laboratorio acá, lo otro es un transcurrir hasta llegar a eso digamos. Es parte de la devolución también (...) uno dice como ‘a mí me gustaría devolver algo después`. Eso lo veo más en la parte de docencia que en la investigación. La investigación es mi carrera digamos. Sé que puedo dar mucho en la docencia» (H, PE, CB).

Todos hicieron referencia a los mayores niveles de competencia existentes en el extranjero en comparación con lo que sucedía a la hora del retorno en Argentina. Esta experiencia presenta dos caras en los relatos. Por un lado, apareció como la posibilidad de «dar un salto» en la propia carrera científica (en términos de publicaciones, de contactos y de apertura a problemas de investigación propios) y, por el otro, como un límite. En la medida en que tenían que medirse con muchos otros que estaban igual o mejor en términos de capital científico, las posibilidades de inserción laboral se volvieron más limitadas una vez concluida la etapa posdoctoral. Esta situación se exacerbó en el caso de las científicas que fueron madres, dado que el tiempo dedicado a la propia carrera disminuyó frente a la llegada de los hijos, por lo que, frente a la dificultad de lograr un puesto permanente en el extranjero o de estar en el mismo nivel de competencia, la opción del retorno a un lugar que reconocía en términos económicos y simbólicos la propia trayectoria se posicionó como una elección atractiva y viable.

«-Para conseguir subsidios tenés que tener publicaciones de buen nivel, con lo cual conseguir publicaciones de buen nivel es más fácil allá por lo que te digo. Por la cantidad de recursos que tenés. A lo mejor un trabajo que acá te llevaría dos años allá acá lo hacés en uno, y un posdoctorado de cinco años acá quizás podés hacer 30 4 papers, en cambio allá podés hacer 7 u 8 . Eso es lo que va haciendo la diferencia, lamentablemente. El número te lo hace. Y allá hay cosas que, más allá de los recursos, andan más fácil» ( $\mathrm{H}, \mathrm{PE}, \mathrm{CB})$. 
«-Nacieron mis hijos y yo quería seguir investigando. Hasta el posdoctorado todo bien, luego tenía que empezar a competir con varones y la competencia es feroz. Hay personas como los chinos que trabajan I4 horas sin parar y con dos hijos llegar a eso es imposible. Para buscar un cargo fijo tenés que trabajar muchas horas y las horas de cuidado de mis hijos no me lo permitían» (M, $\mathrm{PE}, \mathrm{CE})$.

Si bien las migraciones de científicos se consideran «privilegiadas», en los relatos los entrevistados aparecieron -principalmente en el contexto europeo- dos lecturas acerca de la propia integración en el país receptor. Por un lado, la de aquellos que consideraron que gracias a la visa que les otorgaban estaban integrados. Por el otro, la mirada de los que sufrieron las consecuencias de las restricciones en materia migratoria (principalmente para los acompañantes) o la cerrazón cultural y poca disponibilidad de espacios laborales para insertarse una vez concluida la etapa formativa.

«-Me dieron una visa de científico. Lo primero que me dijeron allá es que la cuide porque era una visa muy preciada porque viste que hay mucho tráfico y robo de visas. Así que yo la guardé en la caja fuerte y no la saqué nunca más (...) Tuve una visa de las más altas, quiero decir, yo no era un ocupa en Francia» (H, PE, T).

«-Y lo negativo creo que la integración en la cultura alemana es muy difícil, me costó muchísimo, me sentí siempre un extranjero, a pesar de haber estado 7 años y medio. Los alemanes son bastante particulares (...) Además tampoco había mucho lugar. No son muy abiertos a que los extranjeros se incorporen a la academia alemana. Sí son abiertos a la hora de becar para que uno vaya (...) pero lograr establecerse o radicarse es bastante complicado» (H, DE, CS).

En síntesis, los que se fueron a hacer el doctorado completo estuvieron el doble de tiempo en el extranjero que aquellos que fueron a realizar un posdoctorado. Más allá de esta diferencia, todos reconocen que una vez finalizado el posdoctorado y llegado el momento de buscar un trabajo estable -que no sea una beca o un contrato a término- la competencia se volvió creciente y más difícil de sobrellevar. De ahí que, frente a la posibilidad de poder ingresar a la carrera de investigador en Argentina, la idea del retorno se haya tornado más atractiva. Si bien las cuestiones familiares y afectivas adquieren un peso en el discurso, lo central en la decisión 
es la garantía de llegar a un lugar en el que no sólo se puede continuar la carrera académica, sino también dar un «salto» ya sea por el nivel de independencia a la hora de trabajar como el reconocimiento social de la propia actividad.

\section{EL RETORNO Y LA REINSERCIÓN ACADÉMICA}

Al momento de las entrevistas, los retornados no sólo eran investigadores de CONICET, sino también docentes universitarios. Actividad, esta última, que realizan como una tarea que se encuentra en segundo plano en comparación con la principal que es la de investigar. La docencia se percibe como una forma de "devolver», pero no como una actividad asociada a la ciencia la que, para ellos, pasa por otro lado. No es el objetivo de este trabajo centrarnos en el modo en el que conciben la actividad científica los retornados, de todos modos, destacamos la necesidad de profundizar en otros trabajos acerca de la separación que se percibe entre las dos actividades troncales del mundo académico.

En términos generales, los entrevistados se reinsertaron en un lugar de trabajo en el que habían transitado previamente alguna instancia académica (licenciatura, doctorado o posdoctorado), salvo dos de ellos, en los que la decisión de establecerse en Santa Fe estuvo guiada principalmente por un nexo familiar (ellos o sus parejas son oriundos de la ciudad) más que a la vinculación previa con la UNL. Si bien uno de los entrevistados había dado clases allí durante un período de tiempo, no transcurrió ninguna de las etapas formativas nombradas. Esta desvinculación previa marca una diferencia entre quienes no tenían un nexo previo y quienes sí, a la hora del retorno, ya que los primeros muestran mayores dificultades que los segundos a la hora de integrarse a los grupos o espacios existentes.

«-Hay temas que están de moda en ciertos países y no en otros. Por ejemplo, el tema que yo estoy trabajando no está de moda en Argentina [...] En Argentina no se trabaja lo que yo estoy haciendo. Hay solo una persona en Buenos Aires, pero somos excepciones» (H, DE, CS).

«-Ahora necesitamos gente para el grupo de trabajo. Todavía no hay un grupo humano, acá es difícil competir con los grupos más grandes. Nos pasó de formar varios doctores que se terminaron yendo a los otros grupos» ( $\mathrm{M}, \mathrm{PE}, \mathrm{CE}$ ). 
«-Por eso te digo que de repente me encontré con que tenía que hacer mi investigación y no tenía dónde hacerla. [...] Una vez inicié los trámites para que me dieran una oficina y era todo muy complicado. Me dieron demasiados papeles, demasiadas cosas. [...] Y me encontré con que tenía que trabajar en mi departamento, y trabajar en tu departamento es un problema» (H, DE, CS).

Ambos volvieron a un lugar en el que no tenían una historia anterior en cuanto a líneas de trabajo o nexos personales, por lo que articular con espacios laborales o recorridos de investigación existentes se hizo más complicado. Estas experiencias demuestran que quienes retornan en esas condiciones, requieren de una inversión mayor en términos de construcción de capital social y de capital científico en el contexto de arribo que el hecho de haber estado en el extranjero no les aporta automáticamente, sino que, en algunos casos, les resta. Esta pérdida de capitales se observa con claridad en la comparación con quienes sí tenían una historia previa y contaban con nexos personales y/o con la posibilidad de articular sus propias líneas de trabajo con las existentes.

«-De hecho, pude venir por el apoyo persona a persona que había, te estoy hablando de mi director como investigador que hizo todos los trámites para que se pueda dar esto del PIDRI ${ }^{20}$ y de la entrada a CONICET, toda la parte administrativa. Era difícil por ahí encontrar el dato preciso, más en el 2010 o 20II. La página de Internet no estaba tan bien hecha y yo me la pasaba llamando a CONICET y nadie me daba respuestas, y desde acá me ayudaron mucho» (H, DE, CE).

«Lo primero que hice cuando volví es (...) tratar de a poco de elaborar mis líneas de investigación. Yo me incorporé a un laboratorio que hacía otra cosa. Entré como un asociado en el laboratorio que no trabajaban mis mismos temas. Entonces yo empecé a tratar de a poquito de armar mis líneas de investigación (Н, РE, Св).

20 Proyecto de investigación y desarrollo para la radicación de investigadores (PIDRI) en http://www. raices.mincyt.gob.ar/acciones-repatriacion-pidri.php (último ingreso: 16/11/2018). 
En lo que refiere al capital social adquirido durante la estadía en el extranjero hay dos tipos de experiencias. Una que reconoce que una vez transcurrido un tiempo se comienzan a agotar los vínculos y las relaciones y otra que asume que se mantienen y pueden crecer con el tiempo.

«-Las conexiones se desvanecen con el tiempo, durante dos o tres años publiqué con gente de allá, pero eso con el tiempo se fue perdiendo el contacto» ( $\mathrm{M}, \mathrm{PE}, \mathrm{CE})$.

«-Si, uno de los subsidios (...) es para mantener proyectos en conjunto con el que era mi exjefe. Con lo cual lo hacemos constantemente. Y tengo colaboraciones con otros chicos, con una gente de Francia (...) con un chico de Dinamarca, ahora estamos empezando a hacer algo juntos también» ( $\mathrm{H}, \mathrm{PE}, \mathrm{CB})$.

Si bien los nexos establecidos se valoran como un recurso variable, el hecho de haber tenido una experiencia académica en el extranjero es asumida como un elemento que los distingue de otros. Esa valoración se hace, en la mayoría de los casos, en comparación con aquellos compañeros de trabajo que hicieron su carrera en el mismo lugar y que no tuvieron una estadía de estudio o perfeccionamiento en el extranjero.

«-[El que se movió] fue buscando su carrera y fue proponiendo sus proyectos, y fue gestándose una carrera y fue eligiendo en base a su crecimiento, [a diferencia de] aquel que se fue quedando siempre en el mismo lugar haciendo lo que sabe hacer. Creo que eso genera necesariamente un valor agregado. Después esa persona puede ser mejor o peor, pero creo que como promedio genera un valor agregado. ¿En qué? En la experiencia que tiene esa persona, en los contactos, en la forma en que hace ciencia» ( $\mathrm{H}, \mathrm{PE}, \mathrm{CB})$.

«-No sólo porque ves cómo funciona la ciencia afuera sino también porque madurás como persona» (H, $\mathrm{PE}, \mathrm{T})$.

Estas citas captan las valoraciones que se hicieron al respecto y que se dividieron en dos tipos. Una que asume que tener una experiencia en diferentes lugares (en el mismo país o en el extranjero) contribuye a tener «un valor agregado» a la hora de hacer ciencia: gracias al conocimiento de diferentes ámbitos y contextos laborales, 
de otros objetos de investigación y formas de abordarlos y en los contactos que se adquieren. Y la otra, por su parte, que reconoce que tiene una incidencia en la propia persona: la madura, la forma, le otorga otras experiencias de vida, etc. Siempre en comparación con aquellos que no han pasado por esa experiencia de cambiar de contexto de trabajo (en el país o en el extranjero).

Es de destacar, la diferencia que existe entre aquellos que retornaron entre $200 \mathrm{I}$ y 2004 y los que lo hicieron con posterioridad. Los primeros refieren al ingreso a la carrera a investigador como una meta alcanzada en un contexto de vacantes limitadas y escasas, mientras que, los segundos, dan cuenta del ingreso y del retorno como un trámite que tuvo respuestas rápidas y facilidades, por ejemplo, en la mudanza internacional.

«- Yo me la jugué que con ese tremendo antecedente de una beca posdoctoral en la Sorbona iba a ingresar a la carrera. Y me salió bien. Pero te estoy hablando de que ingresé a carrera en el 2002 y la pedí en el 200I. Era uno de cada rooo (...) Yo los veo a los chicos ahora que con justa razón se quejan de que bajó de 900 a 450 pero en ese momento te estoy hablando de una situación de guerra» (H, PE, T).

«- Lo positivo fue que me presenté al programa de reinserción. Vamos a todos los puntos positivos. El programa salió relativamente rápido, es decir que en uno o dos meses me contestaron. No tuve que esperar tanto tiempo. Eso fue muy positivo. Muy positivo fue que me pagaran absolutamente todo para volver, no tengo ninguna queja porque a mí me pagaron hasta el último centavo de lo que me costó la mudanza internacional. Me pagaron hasta el último peso a mí y a mi mujer. Y cuando volví a Argentina inmediatamente me dieron la beca posdoctoral de CONICET y el ingreso a carrera me salió en poco tiempo (...) Ingresé a carrera en el 2OI2» (H, DE, CS).

En síntesis, hay una diferencia entre aquellos que habían transitado por alguna instancia formativa en la UNL y los que no, en tanto para estos últimos la reinserción se volvió más ardua en términos de construcción de capital social y científico. Todos asumen que el hecho de haber tenido una experiencia formativa en el extranjero es un recurso favorable y que los distingue de quienes permanecieron en el mismo lugar: sea para la competencia académica sea como «experiencia de vida». Por último, se observó una diferencia marcada entre los que volvieron antes del 2004 y los que lo hicieron con posterioridad, en tanto los primeros muestran la dificultad del 
retorno/ingreso a carrera de investigador en el contexto en el que se encontraba el país y CONICET en esos años, y, los segundos, solo mencionan las facilidades de los trámites y las ayudas extras al momento de decidir la vuelta a Argentina.

\section{PARA CONCLUIR}

A lo largo de estas páginas observamos que las trayectorias académicas de los que retornaron a Santa Fe para ingresar a la carrera de investigador (antes y después de la implementación del Programa de Repatriación) se encuentran condicionadas por: la experiencia académica previa en el lugar de retorno, el género y los incentivos para el retorno ligados a las políticas públicas de ciencia y tecnología.

En lo que refiere a la experiencia académica, si bien se observó que el hecho de haber realizado el doctorado completo en otro país influyó tanto en el período de tiempo que duró la migración como en la edad de finalización del ciclo y del retorno, la principal diferencia se identificó entre aquellos que habían transitado alguna instancia formativa (licenciatura y/o doctorado) en la UNL antes de partir y quienes no. Los primeros tuvieron menos dificultades a la hora de integrarse a los grupos y espacios existentes que los segundos.

En torno al género se reconocieron dos particularidades. La primera ligada a las características de la partida en pareja: mientras que los varones entrevistados emigraron con parejas que no se dedicaban a la actividad científica y lograron acomodar sus actividades (laborales y/o de estudio) al lugar elegido por aquel, por el contrario, las mujeres emprendieron la partida junto con su pareja -que también es científico- a una ciudad consensuada en función de los intereses y posibilidades de investigación de ambos. La segunda, por su parte, se vincula al evento de la llegada de los hijos. Si bien todos los entrevistados hicieron mención de la mayor competencia experimentada en los ámbitos de académicos de los países de emigración en comparación con lo vivido en Argentina, sólo las mujeres científicas con hijos dieron cuenta de la imposibilidad de estar a la altura de esta en el extranjero, debido a que el tiempo dedicado a los hijos produjo una disminución del tiempo dedicado al trabajo.

Las trayectorias académicas aquí estudiadas en términos generales son lineales. Es decir, no existe un desfasaje entre el tiempo normativo de cada etapa y los tiempos personales en que cada investigador atravesó la misma (licenciatura, 
doctorado, ingreso a carrera). Si bien ciertos eventos (nacimientos y separaciones) aparecen como relevantes en el relato de cada uno de los entrevistados a la hora de pensar en volver, se reconoció que los incentivos para el retorno (principalmente la posibilidad de ingresar a la carrera de investigador) fueron centrales. Es por eso que aquellos que regresaron a Argentina antes del 2004 -cuando todavía dicha posibilidad era más difícil y limitada- evidenciaron que si no ingresaban a CONICET hubieran buscado continuar la carrera académica en otro país, mientras que los que lo hicieron luego de ese ańo, no sólo experimentaron el ingreso como un trámite entre otros, sino la posibilidad de dar un salto en términos de capital científico frente a un contexto internacional de la ciencia altamente competitivo.

\section{Referencias bibliográficas}

ABBOTT, ANDREW (2001): Times matters. On theory and method, Chicago, The University of Chicago Press.

BOURDIEU, PIERRE ([1976] 1994): «El campo científico», en: Redes, n 2, Universidad Nacional de Quilmes.

BOURDIEU, PIERRE (2002): Poder, derecho y clases sociales, Bilbao, Desclée de Brouwer. BOURDIEU, PIERRE (2002): La distinción. Criterios y bases sociales del gusto, Madrid, Taurus.

COLLER, XAVIER (2005): Estudios de casos, Madrid, CIS.

CONICET (2017): «Descripción». Disponible en: http://www.conicet.gov.ar/conicet-descripcion/ (último ingreso: 16/11/2018).

DUBAR, CLAUDE (1998): «Trajetórias sociais e formas identitárias: alguns esclarecimentos conceituais e metodológicos», en: Educação \& Sociedade, vol. $19, \mathrm{n}^{\circ} 62$.

FLORES, PATRICIA B. (2011): «Los flujos de movilidad internacional de estudiantes y graduados universitarios argentinos en el contexto de inter-

nacionalización de la educación superior», en: L. Luchilo (coord.), Más allá de la fuga de cerebros. Movilidad, migración y diásporas de argentinos calificados, Buenos Aires, Eudeba.

INDEC (2010): Censo. Disponible en: http:// www.indec.gov.ar/censos_total_pais.asp?id tema_1 =2\&id_tema_2 $=41$ \&id_tema_3 $=135 \& \mathrm{t}=3 \& \mathrm{~s}=1 \& \mathrm{c}=2010$ (último ingreso: $16 / 11 / 2018)$.

KREIMER, PABLO (2006): «¿Dependientes o integrados? La ciencia latinoamericana y la nueva división internacional del trabajo», en: Nómadas, n 24. LOZA, ASCENCIO Y GANDINI, LUCIANA (2009): La emigración de recursos humanos calificados desde países de América Latina y el Caribe. Tendencias contemporáneas y perspectivas, Sistema Económico Latinoamericano y del Caribe (SELA). Disponible en: http://rimd.reduaz.mx/ secciones_documentos/859T023600003722-0(último ingreso: 16/11/2018).

LUCHILO, LUCAS (2007): «Migración de retorno: el caso argentino", en: Centro Redes, Documento 
de Trabajo $n^{\circ}$ 39. Disponible en: http://www. centroredes.org.ar/files/documentos/Doc.Nro39. pdf (último ingreso: 16/11/2018).

LUCHILO, LUCAS (2011): «Entre los mercados y las políticas: la dinámica reciente de la movilidad y migración internacional de recursos humanos en ciencia y tecnología», en: L. Luchilo (coord.), Más allá de la fuga de cerebros. Movilidad, migración y diásporas de argentinos calificados. Buenos Aires, Eudeba.

MAHROUM, SAMI (1999): Highly Skilled Globetrotters: The International Migration of Human Capital, París, DSTI/STP/TIP.

MAXWELL, JOSEPH (1996): Qualitative research design. An Interactive Approach. Thousand Oaks, California, Sage.

MERA, CAROLINA Y HALPERN, GERARDO (2011): «Migraciones internacionales: repensando las ciudades y sus políticas», en: Revista Latina de Sociología, $\mathrm{n}^{\circ} 1$.

MEYER, JEAN B, KAPLAN, DAVID Y CHARUM, JORGE (2001): «El nomadismo científico y la nueva geopolítica del conocimiento», en: Revista Internacional de Ciencias Sociales, UNESCO, nº 168.

MINCYT (2015): Programa Raíces. Una política de Estado, Buenos Aires.

MINCYT (2015): Indicadores de Ciencia y Tecnología argentina 2013, Buenos Aires, Año 17, julio de 2015. Disponible en http://indicadorescti.mincyt. gob.ar/documentos/indicadores_2013.pdf (último ingreso: 16/11/2018).

MINCYT (2017): Lineamientos para una política de investigación fundamental, Secretaría de Planeamiento y Políticas en Ciencia, Tecnología e Innovación Productiva, junio 2017, en: http:// www.mincyt.gob.ar/planes-nacionales/lineamientos-para-una-politica-en-investigacion-fundamental-12959 (último ingreso: 16/11/2018). PELLEGRIN0, ADELA (2001): «Drenaje, movilidad, circulación: nuevas modalidades de la migración calificada», en: Actas del Simposio sobre Migración de las Américas, San José de Costa Rica, CEPAL-OIM, 4-6 de septiembre, publicado en: Notas de Población, nº 73.

RAÍCES (2017): «Red de Argentinos Investigadores y Científicos residentes en el exterior», en: http:// www.raices.mincyt.gob.ar/acciones-repatriacionpidri.php (último ingreso: 16/11/2018).

HERNÁNDEZ SAMPIERI, ROBERTO; FERNÁNDEZ COLLADO, CARLOS Y BAPTISTA LUCIO, PILAR (2014): Metodología de la Investigación, México, McGraw Hill.

SAUTU, RUTH (COMP.) (1999): El método biográfico. La reconstrucción de la sociedad a partir del testimonio de los actores, Buenos Aires, Editorial Belgrano.

STAKE, ROBERT (1995): «Case studies», en: N.K. Denzin y Y.S. Lincoln, Handbook of Qualitative Research, California, Sage.

UNESCO (2012): Atlas mundial de la igual de género en la educación, París, Ediciones Unesco.

UNIÓN DEL PERSONAL CIVIL DE LA NACIÓN DE SANTA FE (2013): «En 10 años aumentó un 75\% la planta de CONICET en Santa Fe», en: http://www.upcnsfe. com.ar/nota/36085-en-10-anos-aumento-un75-por-ciento-la-planta-de-conicet-en-santa-fe (último ingreso: 16/11/2018).

VIDEGAIN, KARINA (2015): Análisis longitudinal del Registro Nacional de Alumnos sobre trayectorias educativas, México, Instituto Nacional para la Evaluación de la Educación. 